\title{
Connection between magnetic interactions and the spin-wave gap of the insulating phase of $\mathrm{NaOsO}_{3}$
}

\author{
Nikolaos Ntallis $\odot,{ }^{1}$ Vladislav Borisov, ${ }^{1}$ Yaroslav O. Kvashnin, ${ }^{1}$ Danny Thonig, ${ }^{2}$ Erik Sjöqvist $\odot,{ }^{1}$ Anders Bergman, ${ }^{1}$ \\ Anna Delin, ${ }^{3,4}$ Olle Eriksson, ${ }^{1,2}$ and Manuel Pereiro $\circledast^{1, *}$ \\ ${ }^{1}$ Department of Physics and Astronomy, Uppsala University, Uppsala 751 20, Sweden \\ ${ }^{2}$ School of Science and Technology, Örebro University, SE-701 82 Örebro, Sweden \\ ${ }^{3}$ Department of Applied Physics, School of Engineering Sciences, KTH Royal Institute of Technology, \\ AlbaNova University Center, SE-10691 Stockholm, Sweden \\ ${ }^{4}$ Swedish e-Science Research Center, KTH Royal Institute of Technology, SE-10044 Stockholm, Sweden
}

(Received 7 May 2021; revised 22 August 2021; accepted 11 October 2021; published 28 October 2021)

\begin{abstract}
The scenario of a metal-insulator transition driven by the onset of antiferromagnetic order in $\mathrm{NaOsO}_{3}$ calls for a trustworthy derivation of the underlying effective spin Hamiltonian. To determine the latter we rely on $a b$ initio electronic-structure calculations, linear spin-wave theory, and comparison to experimental data of the corresponding magnon spectrum. We arrive this way to Heisenberg couplings that are $\lesssim 45$ to $\lesssim 63 \%$ smaller than values presently proposed in the literature and Dzyaloshinskii-Moriya interactions in the region of $15 \%$ of the Heisenberg exchange $J$. These couplings together with the symmetric anisotropic exchange interaction and single-ion magnetocrystalline anisotropy successfully reproduce the magnon dispersion obtained by resonant inelastic x-ray scattering measurements. In particular, the spin-wave gap fully agrees with the measured one. We find that the spin-wave gap is defined from a subtle interplay between the single-ion anisotropy, the Dzyaloshinskii-Moriya exchange, and the symmetric anisotropic exchange interactions. The results reported here underpin the local-moment description of $\mathrm{NaOsO}_{3}$, when it comes to analyzing the magnetic excitation spectra. Interestingly, this comes about from a microscopic theory that describes the electron system as Bloch states, adjusted to a mean-field solution to Hubbard-like interactions.
\end{abstract}

DOI: 10.1103/PhysRevB.104.134433

\section{INTRODUCTION}

In the context of correlated electronic systems, $5 d$ oxides hold a distinct position, realizing the peculiar regime where Coulomb interactions (the Hubbard $U$ in particular), the $d-d$ hopping matrix elements (referred to as $t$ 's), and the spin-orbit coupling (SOC, $\lambda$ ) have similar magnitudes $(\lambda \sim t \sim U$ ). In contrast, in $3 d$ and $4 f$ based magnets, it is possible to identify a smallest energy scale, since the SOC defines a much smaller energy scale in $3 d$ transition-metal compounds $(\lambda \ll t<U)$, and the $4 f-4 f$ hopping is minute in rare-earth systems $(t \ll$ $\lambda<U)$.

The SOC in iridates and osmates, for example, is of order $0.5-0.6 \mathrm{eV}$. In addition, the $5 d$ orbitals, being much more extended than, e.g., the rare-earth $4 f$ states, cause the $5 d$ electrons on neighboring ions to interact much more effectively. In other words, reasonably well-localized magnetic moments can still be in place but ligand-mediated superexchange is greatly enhanced as compared to in, e.g., $4 f$ insulators. Combined with the strong SOC, it may generate

\footnotetext{
${ }^{*}$ Corresponding author: manuel.pereiro@physics.uu.se
}

Published by the American Physical Society under the terms of the Creative Commons Attribution 4.0 International license. Further distribution of this work must maintain attribution to the author(s) and the published article's title, journal citation, and DOI. Funded by Bibsam. highly anisotropic intersite magnetic interactions, with the remarkable situation encountered in $t_{2 g}^{5}$ iridates of having either antisymmetric Dzyaloshinskii-Moriya (DM) [1,2] or symmetric Kitaev $[1,3,4]$ effective coupling parameters that are even larger than the isotropic Heisenberg constant.

Since $t \sim U$, metal-insulator transitions (MIT's) may also occur. Extensively discussed in this respect is the osmium oxide perovskite compound $\mathrm{NaOsO}_{3}$. However, rather than a Mott MIT, the scenario of a Slater insulator transition has been proposed for $\mathrm{NaOsO}_{3}$ [5-9], where the formation of antiferromagnetic (AFM) order is opening the insulating gap.

In this frame of reference, detailed knowledge of the underlying magnetic interactions is crucial. Here we shed light on this matter by means of ab initio calculations and subsequent atomistic spin-dynamics simulations employing the derived effective magnetic interactions. This method has its origin in the seminal paper of Ref. [10], where it was shown that even for materials with itinerant electron states, it is possible to map magnetic low energy excitations to an effective spin Hamiltonian, with localized atom-centered spin moments. Several reviews (see Refs. [11,12]) have been published on this topic, and examples have been given on how such ab initio calculations of the Heisenberg exchange can be used to reproduce experimental values of, e.g., the magnon dispersion. The magnon spectra computed in the present paper follow this methodology, and are compared with experimental data as obtained by resonant inelastic x-ray spectroscopy (RIXS) [13]. We hence calculate from $a b$ initio density functional theory (DFT) a Heisenberg exchange of $\approx 4-6 \mathrm{meV}$, that 
(a)

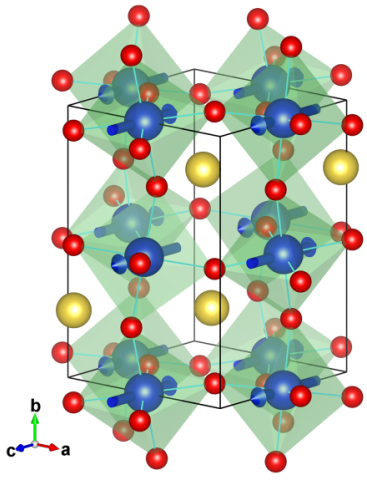

(b)

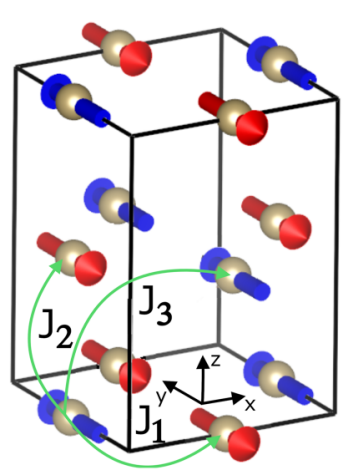

FIG. 1. (a) Pnma crystal structure of $\mathrm{NaOsO}_{3}$. Red and yellow spheres indicate $\mathrm{O}$ and $\mathrm{Na}$ ions, respectively. (b) $G$-type AFM order within the Os sublattice. The nearest-neighbor exchange paths (within the $a c$ plane and along the $b$ axis) are highlighted.

can be easily reconciled with the bandwidth of the magnon spectrum, $\approx 80 \mathrm{meV}$, reported on the basis of RIXS measurements [13]. However, our analysis, as detailed below, shows that a single-ion anisotropy, the DM interaction, Heisenberg exchange, as well as the symmetric anisotropic exchange interaction are large, and necessary for theory to reproduce the magnon spectrum of $\mathrm{NaOsO}_{3}$.

\section{DENSITY FUNCTIONAL THEORY CALCULATIONS}

From the structural point of view, $\mathrm{NaOsO}_{3}$ has the octahedral environment of $\mathrm{Os}^{5+} \mathrm{O}_{6}$, so that the electronic configuration is $5 d^{3}$, suggesting that the $t_{2 g}$ band is half filled. Moreover, this compound shows Curie-Weiss metallic nature and abruptly but continuously turns to an AFM insulator at $410 \mathrm{~K}$ [5]. The structural, electronic, and magnetic properties of bulk $\mathrm{NaOsO}_{3}$ are studied theoretically using DFT [14,15] within the generalized-gradient approximation in the PerdewBurke-Ernzerhof parametrization [16]. Electronic properties are calculated within the all-electron full-potential fully relativistic approach, with linear muffin-tin orbitals as basis functions in both local density approximation (LDA) $+U$ and local spin-density approximation (LSDA) $+U$ approximations, as implemented in the RSPT electronic structure code [17-19], with the $U$ values ranged from 0 to $4 \mathrm{eV}$.

Figure 1(a) shows the experimental crystal structure of $\mathrm{NaOsO}_{3}$. The cell dimensions are $a=5.3842 \AA, b=5.3282$ $\AA$, and $c=7.5804 \AA$. The magnetic ground state for all used values of $U$ has a $G$-type AFM ordering, as shown in Fig. 1(a). In Fig. 2, the spin, orbital, and total magnetic moment per Os atom is plotted with respect to the $U$ value for the different approximations, i.e., $\mathrm{LDA}+U$ and $\mathrm{LSDA}+U$. The two approaches produce different values of the magnetic moments as function of $U$. Notably, the LDA $+U$ approximation would produce a zero magnetic moment for Os atoms by decreasing $U$ down to $2 \mathrm{eV}$, according to Fig. 2. This result is natural, since all exchange splitting in this level of approximation is in the static Hubbard-type interaction, while the spin-density functional by construction provides no exchange splitting. In the limit of finite $U$, where both of the approximations produce almost the same orbital moment, the difference on the spin part is close to $0.35 \mu_{\mathrm{B}}$ per atom. Also, one can reproduce
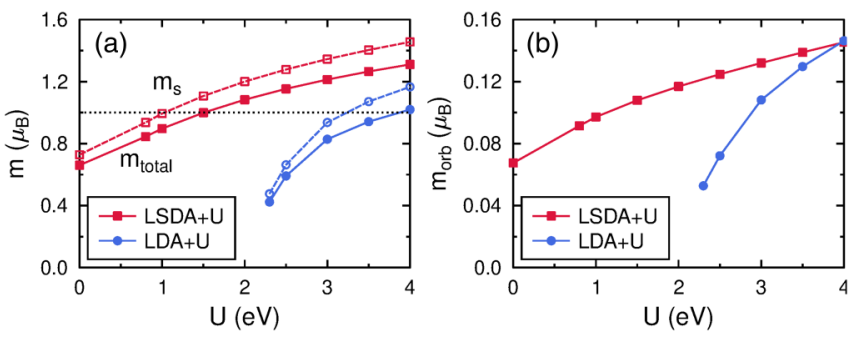

FIG. 2. Calculated total (solid symbols) and spin (unfilled symbols) magnetic moments in (a) together with orbital moments (opposite to the spin moments) per atom of $\mathrm{NaOsO}_{3}$ in (b). The moments are plotted for different values of $U$ and for LSDA $+U$ and $\mathrm{LDA}+U$ implementations with fixed $J_{H}=0.6 \mathrm{eV}$. The dotted line represents the magnetic moment measured in Ref. [6].

the experimental values of the Os moment $\left(\approx 1 \mu_{B}\right)$ for both approximations, albeit for different values of $U$.

It is clear from these results that $\mathrm{NaOsO}_{3}$ is not following a simple picture expected for the half-filled $t_{2 g}$ states with three electrons. In fact, the occupations of Os $5 d$ states are 2.45 (spin-up) and 1.52 (spin-down), as provided by our LSDA $+U$ calculations with $U=0.8 \mathrm{eV}$ and $J_{H}=0.6 \mathrm{eV}$. The strong deviation from the half-filled case is due to the hybridization effects between the Os $5 d$ and oxygen $2 p$ states and the band formation for the Os $5 d$ states.

Different types of magnetic interactions, such as the Heisenberg and DM interaction, were evaluated for the $G$-type antiferromagnetic reference state based on the fully relativistic generalization [20] of the Lichtenstein-Katsnelson-AntropovGubanov formula [10], which we have recently applied to different correlated systems [21]. The purpose of this is to map the electronic system onto a generalized classical Heisenberg model:

$$
H=-\sum_{i \neq j} e_{i}^{\alpha} \hat{J}_{i j}^{\alpha \beta} e_{j}^{\beta}, \quad \alpha, \beta=x, y, z,
$$

where the unit vectors $\mathbf{e}_{i}$ indicate the direction of local spins and the fully relativistic exchange tensor $J_{i j}^{\alpha \beta}$ contains contributions from the Heisenberg exchange as well as the DM interaction $\hat{D}$ and the symmetric anisotropic exchange $\hat{\Gamma} \mathrm{de}-$ fined by

$$
\begin{aligned}
& D_{i j}^{z}=\frac{1}{2}\left(J_{i j}^{x y}-J_{i j}^{y x}\right), \\
& \Gamma_{i j}^{z}=\frac{1}{2}\left(J_{i j}^{x y}+J_{i j}^{y x}\right),
\end{aligned}
$$

and similar expressions for the $x$ and $y$ components.

According to Fig. 2, the values of $U$ which produce the spin magnetic moments closest to the experimental ones are $U \approx 1.0 \mathrm{eV}$ for LSDA $+U$, similarly to Refs. [22-24], and $U \approx 3.0-3.5 \mathrm{eV}$ for $\mathrm{LDA}+U$. Figure 3 shows the calculated magnetic interaction strengths with respect to the atomic distance. All interaction strengths decay quite fast with respect to distance, indicating that the major interactions to be considered are the first and second order ones. The LDA $+U$ approximation (with $U=3.5 \mathrm{eV}$ ) produces Heisenberg exchange interaction that is roughly twice the magnitude compared to the LSDA $+U$ data (with $U=0.8 \mathrm{eV}$ ). Similarly the LDA $+U$ calculation results in a value of $D$ that 

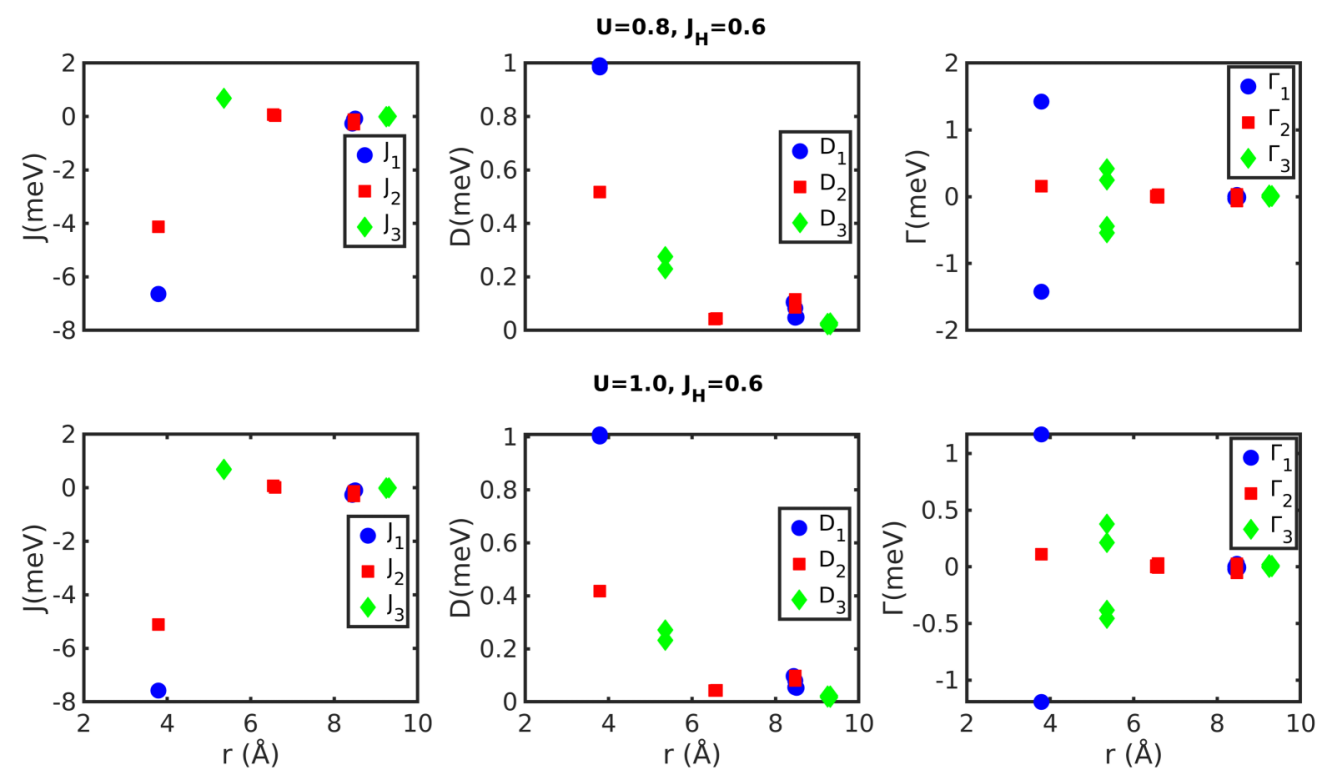

FIG. 3. Calculated interaction strengths $J+D+\Gamma$ with respect to the atomic distance for $U=0.8 \mathrm{eV}, J_{H}=0.6 \mathrm{eV}$ (top panel) and $U=1.0 \mathrm{eV}, J_{H}=0.6 \mathrm{eV}$ (bottom panel). All interactions are marked following the prescription $1 \rightarrow 3$ given in Fig. 1.

is approximately three or four times larger than the one for LSDA $+U$ approximation. In order to assess the quality of the exchange interactions we used them to evaluate adiabatic magnon spectra, that can be compared to experimental results (see discussion below). We note, however, already here that such an analysis reproduces experimental results quite poorly from a theory based on LDA $+U$ (see Supplemental Material for further details on the $\mathrm{LDA}+U$ results [25]), while $\mathrm{LSDA}+U$ calculations give reasonable results. For this reason we focus in the rest of this paper on results from LSDA $+U$ calculations.

Despite the same magnetic pattern along the $x$ and $z$ directions and very similar distances $\left(d_{x}=3.787 \mathrm{~A}\right.$ and $d_{z}=$ $3.790 \mathrm{~A}$ ), the Heisenberg exchange interactions $J_{1 x}$ and $J_{1 z}$ are markedly different. This could be related to the magnetic anisotropy which aligns the magnetic moments and the Néel vector preferably along the $y$ direction and to the structural anisotropy due to the oxygen octahedra rotation and tilting. It is interesting that the $J_{1 z} / J_{1 x}$ ratio increases as a function of the correlation strength $U$ from 0.62 at $U=0.8 \mathrm{eV}$ to 0.85 at $U=4 \mathrm{eV}$. This may suggest that the system becomes magnetically more isotropic for stronger correlations where the system is more insulating, based on the calculated value of the electronic band gap. We also notice that the rescaled Heisenberg parameters $J / m_{s}^{2}$, depicted in Fig. S3 in Supplemental Material [25], are far from the $1 / U$ scaling expected for insulators. This can be explained by the fact that correlations in the narrow-gap insulator $\mathrm{NaOsO}_{3}$ are not strong compared to the electron hopping amplitude $t$, so that $t / U$ is not a small parameter and the perturbation theory, which could lead to the $1 / U$ scaling, is not applicable. Interestingly, the nearest-neighbor parameters $J_{1} / m_{s}^{2}$ increase as functions of $U$ and reach a plateau around $U=2.0 \mathrm{eV}$. On the other hand, the DM interaction shows a nonmonotonous variation of its $y$ component, which is dominating for the bonds along the $x y$ directions, with a maximum around $U=1 \mathrm{eV}$ followed by a decreasing trend for larger $U$ values. The rescaled DM interaction $\left(D_{1 y} / m_{s}^{2}\right.$ in Fig. S3) shows, however, a clear $1 / U$ trend starting from $U=2 \mathrm{eV}$.

Before discussing the magnon spectra, it is worth mentioning that the SOC has a considerable effect on magnetism in $\mathrm{NaOsO}_{3}$. For example, the LSDA $+U$ calculations with $U=0.8 \mathrm{eV}$ and $J_{H}=0.6 \mathrm{eV}$ predict the Os spin moment $m_{s}=0.94 \mu_{\mathrm{B}}$ when SOC is included and $m_{s}=1.09 \mu_{\mathrm{B}}$ when SOC is not included. In those two cases, also the Heisenberg interactions $J_{1}$ are visibly affected by relativistic corrections, as the in-plane and out-of-plane $J_{1}$ parameters equal -6.7 and $-4.1 \mathrm{meV}$ with SOC and -6.3 and $-5.2 \mathrm{meV}$ without SOC. This means that the anisotropy of the $J_{1}$ values is enhanced by the spin-orbit coupling, which naturally is significant in $\mathrm{NaOsO}_{3}$, due to the $5 d$ nature of electronic states that are responsible for the magnetism in this compound.

The numerical values of the exchange interactions are collected in Table I for LSDA $+U$ calculations, while the DM vectors for the nearest-neighbor bonds are shown in Fig. 4. These interactions were used to calculate the magnon spectra, which was evaluated by rewriting Eq. (1), in the form of a bilinear, effective Hamiltonian $\mathcal{H}_{i, j}$ containing the isotropic Heisenberg, the DM, as well as symmetric anisotropic exchange. Adding single-site magnetic anisotropy we end up with the following expression for the full Hamiltonian of the spin system:

$$
\begin{aligned}
\mathcal{H}_{\bmod }^{i, j}= & -J_{i j} \mathbf{S}_{i} \cdot \mathbf{S}_{j}-\mathbf{D}_{i j} \cdot \mathbf{S}_{i} \times \mathbf{S}_{j}-\mathbf{S}_{i} \Gamma_{i j} \mathbf{S}_{j} \\
& -K \sum_{k=i, j}\left(\mathbf{S}_{k} \cdot \mathbf{e}_{k}^{y}\right)^{2},
\end{aligned}
$$

where $\mathbf{S}_{i}$ and $\mathbf{S}_{j}$ are normalized atomic spin moments. The isotropic Heisenberg coupling is given by $J_{i j}$ and $\mathbf{D}_{i j}$ stands for the DM vector, while the tensor $\Gamma_{i j}$ represents the symmetric anisotropic exchange interaction between atomic sites $i$ and $j$. The Heisenberg exchange interaction $J_{i j}$, the norm 
TABLE I. Intersite interaction vectors, effective couplings, and DM vectors for different Os-O-Os bond angles. The units of the coupling parameters are given in $\mathrm{meV}$. The reference system is specified in Fig. 1(b).

\begin{tabular}{lcc}
\hline \hline$\theta_{1}=155.2^{\circ}$ & $U=0.8 \mathrm{eV}$ & $U=1.0 \mathrm{eV}$ \\
\hline$J_{1}$ & -6.64 & -7.58 \\
$\Gamma_{1}$ & 0.62 & 0.57 \\
$\mathbf{r}_{i j}$ & $( \pm 0.5, \pm 0.5,0)$ & $( \pm 0.5, \pm 0.5,0)$ \\
$\mathbf{D}_{1}$ & $\pm(0.04,-0.92,-0.37)$ & $\pm(0.08,-0.92,-0.40)$ \\
$\theta_{2}=153.9^{\circ}$ & $U=0.8 \mathrm{eV}$ & $U=1.0 \mathrm{eV}$ \\
$J_{2}$ & -4.12 & -5.11 \\
$\Gamma_{2}$ & 0.07 & 0.05 \\
$\mathbf{r}_{i j}$ & $(0.0,0.0, \pm 0.5)$ & $(0.0,0.0, \pm 0.5)$ \\
$\mathbf{D}_{2}$ & $U=0.8 \mathrm{eV}$ & $(-0.41,0.07,0.00)$ \\
$\theta_{3}=\emptyset$ & 0.67 & $U=1.0 \mathrm{eV}$ \\
$J_{3}$ & 0.18 & 0.68 \\
$\Gamma_{3}$ & $( \pm 0.5, \pm 0.5, \pm 0.5)$ & $( \pm 0.5, \pm 0.5, \pm 0.5)$ \\
$\mathbf{r}_{i j}$ & $\pm(0.20,-0.01,-0.18)$ & $\pm(0.20,-0.03,-0.18)$ \\
$\mathbf{D}_{3}^{a}$ & $\pm(-0.20,0.01,-0.11)$ & $\pm(0.20,0.00,-0.10)$ \\
$\mathbf{D}_{3}^{b}$ & & \\
\hline
\end{tabular}

of $\mathbf{D}_{i j}$, and the mean value of the $\Gamma_{i j}$ tensor take the values $J_{1},\left|\mathbf{D}_{1}\right|$, and $\Gamma_{1}$ and $J_{2},\left|\mathbf{D}_{2}\right|$, and $\Gamma_{2}$ for bonds 1 and 2 [see Fig. 1(b)], respectively. The orientations of the DM vectors depend on the position in the unit cell (see Fig. 4). The DM vectors $\mathbf{D}_{3}^{a}$ and $\mathbf{D}_{3}^{b}$ for the third-neighbor atoms are slightly different (see Table I and Fig. 4) because the cell dimensions in the $a b$ plane are also slightly different $(a \neq b)$. As regards the magnetic anisotropy, each site possesses an easy-axis orientation directed along the local magnetic $y$ axis $\mathbf{e}_{k}^{y}$. The magnetic anisotropy parameter $[K$ in Eq. (4)] was calculated based on the sum of eigenvalues in the one-shot relativistic calculations using the RSPT code [17-19] where the magnetization axis is oriented along the $x, y$, and $z$ directions. The obtained estimates are $K=2.05 \mathrm{meV}$ for $U=0.8 \mathrm{eV}$ and $J_{H}=0.6 \mathrm{eV}$ while the calculation with $U=1.0 \mathrm{eV}$ and $J_{H}=0.6 \mathrm{eV}$ resulted in $K=2.44 \mathrm{meV}$.
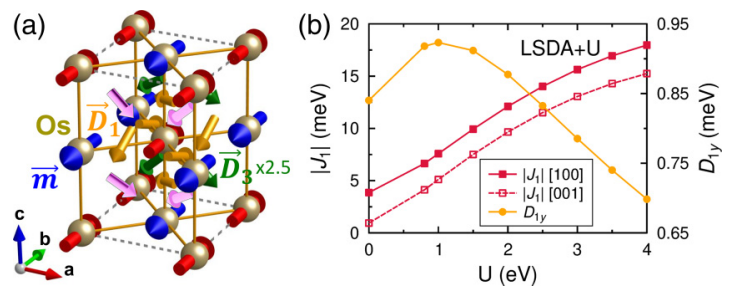

FIG. 4. (a) Calculated DM vectors $\mathbf{D}$ for the nearest-neighbor bonds in $\mathrm{NaOsO}_{3}$ shown by the orange $\left(\mathbf{D}_{1}\right)$, green $\left(\mathbf{D}_{3}^{a}\right)$, and magenta $\left(\mathbf{D}_{3}^{b}\right)$ arrows. The $\mathbf{D}_{3}^{a, b}$ vectors are magnified by the factor of 2.5 for clarity. The $G$-type antiferromagnetic ordering of Os magnetic moments $\mathbf{m}$ oriented along the $y$ direction is depicted by the red and blue arrows. The magnetic interactions were obtained using LSDA $+U$ with $U=0.8 \mathrm{eV}$ and $J_{H}=0.6 \mathrm{eV}$. (b) The nearest-neighbor Heisenberg exchange $J_{1}$ along the [100] and [001] directions and the $y$ component of the DM interaction $\mathbf{D}_{1}$ in the $a b$ plane as functions of the correlation strength $U$.
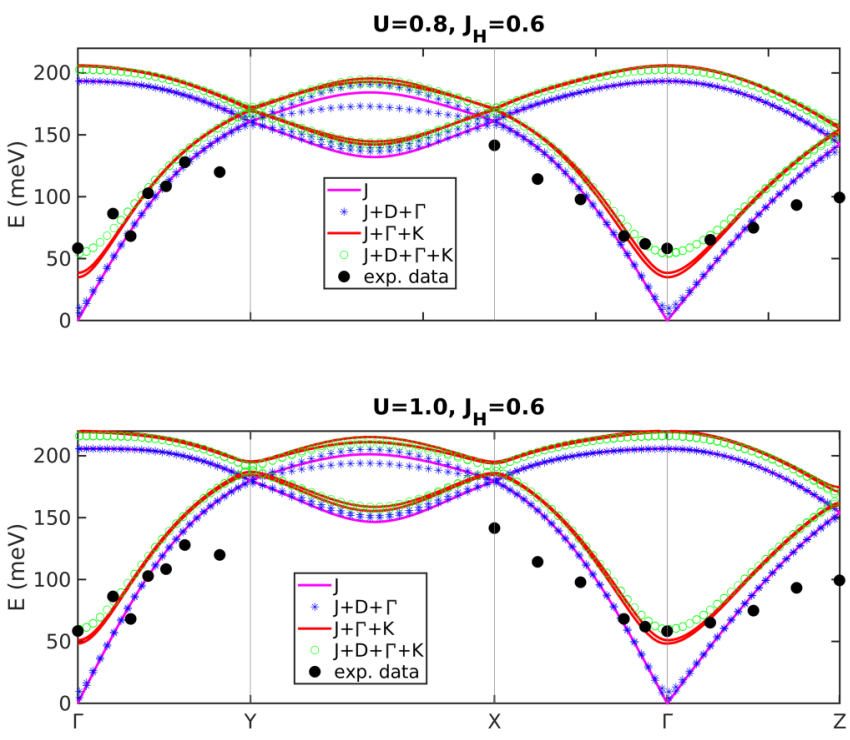

FIG. 5. Adiabatic magnon spectrum of $\mathrm{NaOsO}_{3}$ by progressively adding energy contributions to the effective spin Hamiltonian. We illustrate the cases with contributions given by $J, J+\mathbf{D}+\boldsymbol{\Gamma}, J+\boldsymbol{\Gamma}+K$, $J+\mathbf{D}+\boldsymbol{\Gamma}+K$, for $U=0.8 \mathrm{eV}, J_{H}=0.6 \mathrm{eV}$ from LSDA $+U$ theory (top panel) and $U=1.0 \mathrm{eV}, J_{H}=0.6 \mathrm{eV}$ from LSDA+ $U$ theory (bottom panel). Experimental data provided by RIXS measurements are shown in black dots [13].

\section{LINEAR SPIN WAVE THEORY CALCULATIONS}

In order to validate the parameters calculated by $a b$ initio electronic structure theory, we performed calculations of magnon dispersions, based on a collinear AFM ground state, in the framework of linear spin-wave theory (LSWT) [2] by using the UppASD software [26]. Effective coupling parameters as collected in Table I were utilized, along with a moment as illustrated in Fig. 2(a), from LSDA $+U$ approximation using $U=0.8-1.0 \mathrm{eV}$ and $J_{H}=0.6 \mathrm{eV}$.

In order to understand which interactions dominate the spin-wave gap of $\mathrm{NaOsO}_{3}$, we calculated adiabatic magnon spectra by progressively adding different energy terms as present in Eq. (4) (see Fig. 5). It is evident from the figure that the uniaxial single-ion anisotropy is the term responsible for the spin-wave gap and the value of $U$ that best fit the experimental spin-wave gap is achieved by $U=1.0 \mathrm{eV}$ while for $U=0.8 \mathrm{eV}$ the gap is slightly too low (the magnon excitations are evaluated for a wider range of values of $U$ in the Supplemental Material [25]). Furthermore, the contributions of the DM interaction together with the symmetric anisotropic exchange interaction, even though they are small, are necessary to correctly describe the full experimental spin-wave dispersion. Interestingly, the calculated profile of the magnon spectra for $U=1 \mathrm{eV}$ agrees quite well with the experimental spectra, even though the exchange coupling parameters together with the anisotropy constant differ substantially with respect to the ones suggested from the experimental data in Ref. [13]. A direct fitting procedure of the magnon spectra, with a relatively large number of parameters in a spin Hamiltonian, can agree with experimental data but the parameters of the Hamiltonian obtained from such fittings might be completely off compared to interactions calculated 

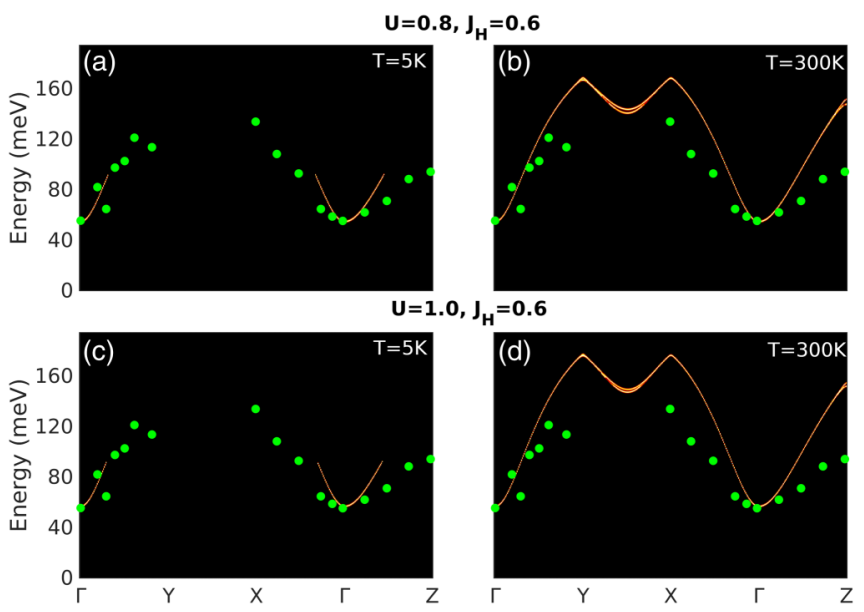

FIG. 6. Dynamical structure factor of $\mathrm{NaOsO}_{3}$ for (a) $U=$ $0.8 \mathrm{eV}, J_{H}=0.6 \mathrm{eV}$ at $T=5 \mathrm{~K}$, (b) $U=0.8 \mathrm{eV}, J_{H}=0.6 \mathrm{eV}$ at $T=$ $300 \mathrm{~K}$, (c) $U=1.0 \mathrm{eV}, J_{H}=0.6 \mathrm{eV}$ at $T=5 \mathrm{~K}$, and (d) $U=1.0 \mathrm{eV}$, $J_{H}=0.6 \mathrm{eV}$ at $T=300 \mathrm{~K}$. All the aforementioned parameters are calculated at the level of LSDA $+U$ theory. Energy is convoluted with a $0.7-\mathrm{meV}$ full width at half maximum Gaussian. Experimental data provided by RIXS measurements are shown by green dots [13].

from $a b$ initio electronic structure theory. This illustrates that $a b$ initio theory is crucial for extracting proper interaction types, strengths, and ranges.

The calculated adiabatic magnon excitation energies are in good agreement with the experimental data reported in Ref. [13], as shown in Fig. 5. However, the adiabatic magnon spectrum is formally calculated at $T=0 \mathrm{~K}$, whereas the experimental measurements are done at room temperature, i.e., $T=300 \mathrm{~K}$. Therefore theory and experiments refer to data obtained at different temperatures. In order to shed more light on this point, we show in Fig. 6 the dynamical structure factor calculated in the framework of LSWT, which is proportional to the differential cross section measured in scattering experiments. It depends on the crystal momentum (q) and energy transfer $(\omega)$ and is defined as a $3 \times 3$ matrix:

$$
S(\mathbf{q}, \omega)=\frac{1}{2 \pi N} \sum_{i, j} e^{i \mathbf{q}\left(\mathbf{r}_{i}-\mathbf{r}_{j}\right)} \int_{-\infty}^{\infty} e^{-i \omega t}\left\langle\mathbf{S}_{i}(t) \mathbf{S}_{j}^{T}(t)\right\rangle d t,
$$

where indices $i, j$ label the atomic positions and $\mathbf{r}_{i}$ is the position vector of atom $i$. $N$ is the number of magnetic atoms in the unit cell and $\mathbf{S}_{i}$ represents a column vector of the $x$, $y$, and $z$ spin operator components. The symbol $\langle\ldots\rangle$ denotes the ensemble average in thermal equilibrium with the environment at temperature $T$. Since we are dealing with magnons, i.e., bosons, $T$ is introduced in the thermodynamic average by using Bose-Einstein statistics, with a Bose factor

$$
n(\omega)=\frac{1}{e^{\hbar \omega / k_{B} T}-1} .
$$

In Figs. 6(a) and 6(c), we show $S(\mathbf{q}, \omega)$ for $\mathrm{NaOsO}_{3}$ using magnetic interactions according to Eq. (4) and Table I at $T=5 \mathrm{~K}$ and in Figs. 6(b) and 6(d) the dynamical structure factor is calculated for $T=300 \mathrm{~K}$, the value of the temperature at which the experimental measurements have been reported in Ref. [13]. The dispersion of the curve in Fig. 6 is naturally the same as in Fig. 5 but additional information is obtained from the intensity, which reveals how likely a scattering event is. Notably, for $T=5 \mathrm{~K}$ only in the vicinity of the $\Gamma$ point does the cross section show a significant intensity indicating that at very low temperatures only these specific modes would be captured. On the other hand, at a temperature of $T=300 \mathrm{~K}$, the two lowest energy branches show a considerable intensity along the $\mathbf{q}$ path in agreement with experimental data. Note here that according to Fig. 5, the first acoustic and first optical branches are very close in energy and thus both of them are activated by increasing temperature. Figure 6 also reveals that at $300 \mathrm{~K}$, the experimental conditions of the RIXS measurements, the experimental apparatus only can capture the acoustic and first optical branches while the additional third and fourth optical branches remain hidden to the experiment. See Supplemental Material for a discussion about the calculated dynamical correlation function by using spin-dynamics simulations.

\section{SUMMARY AND CONCLUSIONS}

In summary, the very rare situation in which $a b$ initio calculations of Heisenberg exchange result in $\lesssim 45$ to $\lesssim 63 \%$ smaller values compared to those obtained through fitting of experimental magnon dispersions by LSWT has led us to a careful analysis of the spin excitation spectrum of the osmate perovskite $\mathrm{NaOsO}_{3}$. To this end, we carried out atomistic spin-dynamics simulations using as input the effective magnetic interactions determined from $a b$ initio calculations. These interactions involved not only Heisenberg but also DM and symmetric anisotropic intersite couplings, plus single-ion spin anisotropies. The effective DM interaction parameters turn out to be sizable, $\approx 14 \%$ of the Heisenberg $J_{1}$ 's for $U=1 \mathrm{eV}$, while the single-ion anisotropies are also robust. Our theoretical results reproduce with good accuracy the observations, albeit with a distinct difference in that the spectra along the $x \rightarrow \Gamma \rightarrow z$ path are slightly steeper than the measured spectra. Moreover, the third and fourth optical branches demonstrated in the theory are not found in the experimental measurements. We propose a mechanism based on thermal fluctuations for why these two modes are hidden from experimental detection. The analysis put forth here differs from that of Ref. [13] in that our calculations provide somewhat smaller magnetic anisotropy, but notably a sizable DM exchange which was not suggested in Ref. [13]. We note that the DM interaction can on general grounds not be neglected in $\mathrm{NaOsO}_{3}$ since the $\mathrm{O}$ ion mediating superexchange between two Os nearest neighbors does not sit at an inversion center and SO interactions are large for the $5 d$ shell. Hence, one should anticipate that the DM interaction is large, which our theoretical calculations confirm. We have demonstrated that the single-ion anisotropy along with DM interaction and to a less extent the symmetric anisotropic exchange interaction clearly have a role in the spin-wave gap of $\mathrm{NaOsO}_{3}$. The contribution of the DM and $\Gamma$ interaction as the microscopic mechanism behind a spin-wave gap is seldom discussed, and $\mathrm{NaOsO}_{3}$ is a unique material in this sense.

While our results for the magnon modes faithfully reproduce the dispersion reported on the basis of RIXS experiments [13], especially close to the zone center, we must 
acknowledge a larger discrepancy between theory and observation for modes close to the zone boundary. This could potentially be caused by excitation mechanisms that go beyond a model of excitations of an extended Heisenberg model with localized atomic moments, where, e.g., electronic and magnetic excitations coexist. Alternatively, the difference could be due to a configuration dependence of the magnetic parameters (Heisenberg exchange and antisymmetric and symmetric anisotropic exchange), that were evaluated from the ground state, and hence would be expected to better reproduce excitations close to the zone center. We also point out that in the regime of observed magnons the optical and acoustic modes are sometimes close in energy, and in order to identify which mode is observed it is important to theoretically analyze the scattering amplitudes. Furthermore, we find that a full account of all interaction parameters is needed in order to reproduce experimental observations of this complex compound.

Finally, we note that it was argued in Ref. [13] that $\mathrm{NaOsO}_{3}$ is a system on the boundary between local-moment and itinerant magnetism, based on the fact that the SOC is very strong with a spin gap of $58 \mathrm{meV}$ around the $\Gamma$ point. Our results can explain experimental magnon spectra by assuming an effective on-site Coulomb repulsion of $U_{\text {eff }}=U-J_{H}=0.4 \mathrm{eV}$ which drives the system out of the purely itinerant regime. In particular, the parameter choice $U \approx 0.8-1.0 \mathrm{eV}$ and $J_{H}=$ $0.6 \mathrm{eV}$ provides a good description of the spin-wave gap and the Os moment compared to the measurements. We would like to emphasize the important role of spin-orbit coupling for this magnetic system, which is indicated by the changes in the calculated magnetic moments and interactions due to SOC. In addition, SOC has to be included in the calculations, since otherwise the DM interaction [see Eq. (2)] and the symmetric anisotropic exchange [see Eq. (3)] are both strictly zero.

\section{ACKNOWLEDGMENTS}

The authors acknowledge financial support from Knut and Alice Wallenberg Foundation through Grant No. 2018.0060. A.D. acknowledges financial support from the Swedish Research Council (VR) through Grants No. 2015-04608, No. 2016-05980, and No. 2019-05304. O.E. also acknowledges support from eSSENCE, Swedish National Infrastructure for Computing (SNIC), the Swedish Research Council (VR), and the European Research Council (synergy grant FASTCORR, Project No. 854843). D.T. acknowledges support from the Swedish Research Council (VR) through Grant No. 201903666. E.S. acknowledges financial support from the Swedish Research Council (VR) through Grant No. 2017-03832. The work of Y.O.K. is supported by the Swedish Research Council (VR) under Project No. 2019-03569. Some of the computations were performed on resources provided by SNIC at the National Supercomputer Center, Linkping University, the PDC Centre for High Performance Computing, KTH, partially funded by the Swedish Research Council through Grant No. 2016-07213 and the High Performance Computing Center North (HPC2N), Umeå.
[1] G. Jackeli and G. Khaliullin, Mott Insulators in the Strong Spin-Orbit Coupling Limit: From Heisenberg to a Quantum Compass and Kitaev Models, Phys. Rev. Lett. 102, 017205 (2009).

[2] R. Yadav, M. Pereiro, N. A. Bogdanov, S. Nishimoto, A. Bergman, O. Eriksson, J. van den Brink, and L. Hozoi, Heavymass magnetic modes in pyrochlore iridates due to dominant Dzyaloshinskii-Moriya interaction, Phys. Rev. Materials 2, 074408 (2018).

[3] Y. Yamaji, Y. Nomura, M. Kurita, R. Arita, and M. Imada, FirstPrinciples Study of the Honeycomb-Lattice Iridates $\mathrm{Na}_{2} \mathrm{IrO}_{3}$ in the Presence of Strong Spin-Orbit Interaction and Electron Correlations, Phys. Rev. Lett. 113, 107201 (2014).

[4] V. M. Katukuri, S. Nishimoto, V. Yushankhai, A. Stoyanova, H. Kandpal, S. Choi, R. Coldea, I. Rousochatzakis, L. Hozoi, and J. van den Brink, Kitaev interactions between $j=1 / 2$ moments in honeycomb $\mathrm{Na}_{2} \mathrm{IrO}_{3}$ are large and ferromagnetic: Insights from $a b$ initio quantum chemistry calculations, New J. Phys. 16, 013056 (2014).

[5] Y. G. Shi, Y. F. Guo, S. Yu, M. Arai, A. A. Belik, A. Sato, K. Yamaura, E. Takayama-Muromachi, H. F. Tian, H. X. Yang, J. Q. Li, T. Varga, J. F. Mitchell, and S. Okamoto, Continuous metal-insulator transition of the antiferromagnetic perovskite $\mathrm{NaOsO}_{3}$, Phys. Rev. B 80, 161104(R) (2009).

[6] S. Calder, V. O. Garlea, D. F. McMorrow, M. D. Lumsden, M. B. Stone, J. C. Lang, J.-W. Kim, J. A. Schlueter, Y. G. Shi, K. Yamaura, Y. S. Sun, Y. Tsujimoto, and A. D. Christianson, Magnetically Driven Metal-Insulator Transition in $\mathrm{NaOsO}_{3}$, Phys. Rev. Lett. 108, 257209 (2012).
[7] Y. Du, X. Wan, L. Sheng, J. Dong, and S. Y. Savrasov, Electronic structure and magnetic properties of $\mathrm{NaOsO}_{3}$, Phys. Rev. B 85, 174424 (2012).

[8] M.-C. Jung, Y.-J. Song, K.-W. Lee, and W. E. Pickett, Structural and correlation effects in the itinerant insulating antiferromagnetic perovskite $\mathrm{NaOsO}_{3}$, Phys. Rev. B 87, 115119 (2013).

[9] I. L. Vecchio, A. Perucchi, P. Di Pietro, O. Limaj, U. Schade, Y. Sun, M. Arai, K. Yamaura, and S. Lupi, Infrared evidence of a Slater metal-insulator transition in $\mathrm{NaOsO}_{3}$, Sci. Rep. 3, 2990 (2013).

[10] A. Liechtenstein, M. Katsnelson, V. Antropov, and V. Gubanov, Local spin density functional approach to the theory of exchange interactions in ferromagnetic metals and alloys, J. Magn. Magn. Mater. 67, 65 (1987).

[11] C. Etz, L. Bergqvist, A. Bergman, A. Taroni, and O. Eriksson, Atomistic spin-dynamics and surface magnons, J. Phys.: Condens. Matter 27, 243202 (2015).

[12] O. Eriksson, A. Bergman, L. Bergqvist, and J. Hellsvik, Atomistic Spin-Dynamics: Foundations and Applications (Oxford University, New York, 2017).

[13] S. Calder, J. G. Vale, N. Bogdanov, C. Donnerer, D. Pincini, M. Moretti Sala, X. Liu, M. H. Upton, D. Casa, Y. G. Shi, Y. Tsujimoto, K. Yamaura, J. P. Hill, J. van den Brink, D. F. McMorrow, and A. D. Christianson, Strongly gapped spin-wave excitation in the insulating phase of $\mathrm{NaOsO}_{3}$, Phys. Rev. B 95, 020413(R) (2017).

[14] P. Hohenberg and W. Kohn, Inhomogeneous electron gas, Phys. Rev. 136, B864 (1964). 
[15] W. Kohn and L. J. Sham, Self-consistent equations including exchange and correlation effects, Phys. Rev. 140, A1133 (1965).

[16] J. P. Perdew, K. Burke, and M. Ernzerhof, Generalized Gradient Approximation Made Simple, Phys. Rev. Lett. 77, 3865 (1996).

[17] J. M. Wills and B. R. Cooper, Synthesis of band and model Hamiltonian theory for hybridizing cerium systems, Phys. Rev. B 36, 3809 (1987).

[18] J. Wills, O. Eriksson, M. Alouani, and D. Price, Full-potential LMTO total energy and force calculations, in Electronic Structure and Physical Properties of Solids (Springer-Verlag, Berlin, 2000).

[19] J. Wills, M. Alouani, P. Andersson, A. Delin, O. Eriksson, and O. Grechnyev, Full-Potential Electronic Structure Method, Springer Series in Solid-State Sciences (Springer-Verlag, Berlin, 2010), Vol. 167.

[20] Y. O. Kvashnin, A. Bergman, A. I. Lichtenstein, and M. I. Katsnelson, Relativistic exchange interactions in $\mathrm{Cr} X_{3}(X=\mathrm{Cl}$, Br, I) monolayers, Phys. Rev. B 102, 115162 (2020).

[21] V. Borisov, Y. O. Kvashnin, N. Ntallis, D. Thonig, P. Thunstrom, M. Pereiro, A. Bergman, E. Sjoqvist, A. Delin,
L. Nordstrom, and O. Eriksson, Heisenberg and anisotropic exchange interactions in magnetic materials with correlated electronic structure and significant spin-orbit coupling, Phys. Rev. B 103, 174422 (2021).

[22] S. Middey, S. Debnath, P. Mahadevan, and D. D. Sarma, $\mathrm{NaOsO}_{3}$ : A high Neel temperature $5 d$ oxide, Phys. Rev. B 89, 134416 (2014).

[23] S. Mohapatra, C. Bhandari, S. Satpathy, and A. Singh, Effect of structural distortion on the electronic band structure of $\mathrm{NaOsO}_{3}$ studied within density functional theory and a three-orbital model, Phys. Rev. B 97, 155154 (2018).

[24] P. Liu, J. He, B. Kim, S. Khmelevskyi, A. Toschi, G. Kresse, and C. Franchini, Comparative ab initio study of the structural, electronic, magnetic, and dynamical properties of $\mathrm{LiOsO}_{3}$ and $\mathrm{NaOsO}_{3}$, Phys. Rev. Materials 4, 045001 (2020).

[25] See Supplemental Material at http://link.aps.org/supplemental/ 10.1103/PhysRevB.104.134433 for further details.

[26] B. Skubic, J. Hellsvik, L. Nordström, and O. Eriksson, A method for atomistic spin dynamics simulations: Implementation and examples, J. Phys.: Condens. Matter 20, 315203 (2008). 